\title{
Computer assessment of interview data using latent semantic analysis
}

\author{
Gregory Dam AND Stefan KaufmanN \\ Northwestern University, Evanston, Illinois
}

\begin{abstract}
Clinical interviews are a powerful method for assessing students' knowledge and conceptual development. However, the analysis of the resulting data is time-consuming and can create a "bottleneck" in large-scale studies. This article demonstrates the utility of computational methods in supporting such an analysis. Thirty-four 7thgrade student explanations of the causes of Earth's seasons were assessed using latent semantic analysis (LSA). Analyses were performed on transcriptions of student responses during interviews administered, prior to $(n=21)$ and after $(n=13)$ receiving earth science instruction. An instrument that uses LSA technology was developed to identify misconceptions and assess conceptual change in students' thinking. Its accuracy, as determined by comparing its classifications to the independent coding performed by four human raters, reached $90 \%$. Techniques for adapting LSA technology to support the analysis of interview data, as well as some limitations, are discussed.
\end{abstract}

Researchers in education have long been faced with the challenge of developing ever more improved methods for assessing students' knowledge. Understanding the variety of conceptions that students bring to instruction is a prerequisite for effecting conceptual change (Carey, 1988; Posner, Strike, Hewson, \& Gertzog, 1982). For this reason, tools that help support assessments of students' understanding are welcomed commodities. Recently there has been much interest in developing computational instruments for evaluating student knowledge. In the long run, the development of such tools serves two main practical goals: to increase the level of rigor and objectivity in student assessment and to improve efficiency by enabling the processing and analysis of large amounts of data with little or no human supervision. Which computational approach is most promising is still an open question, one that depends to a large extent on the kind of data and the goals of the analysis.

In this article, we report the results of experiments in the computational analysis of clinical interview data. Unlike other common methods for assessing students' knowledge, clinical interviews are not driven by tightly controlled scripts or protocols, but are rather unstructured, open-ended, and often discursive in style. The interviewer is encouraged to react to students' responses with digressions, questions, or small impromptu experiments. There are many advantages to using interviews for probing student thinking. In particular, unlike written assessments, which are not flexible enough to trace the complex dynamics of students' understanding, interviews offer a unique way to test hypotheses about what a student is thinking at a given moment (Ginsburg, 1997).

Computational methods have not previously been applied in the analysis of clinical interview data. The clos- est area in which a significant amount of computational work has been carried out is the design of intelligent tutoring systems (ITS; Graesser et al., 2004; Wade-Stein \& Kintsch, 2004). The purpose of an ITS is to replace the human instructor in interactions with one or more students. The most challenging task in doing so is to track each student's understanding by presenting them with questions and evaluating their responses, continually updating an internal model of the student's knowledge and making decisions - for instance, how to present information, at what level to gauge understanding through questioning - on the basis of that model.

Whereas ITS design and our interview analysis share the task of assessing students' knowledge, they differ in a number of important respects. Our goal is to analyze transcripts of interviews that have been completed, not to conduct the interviews. This allows us to avoid two hard problems inherent in ITS design: the need to make decisions in real time based on a dynamically changing model of students' knowledge, and the need to generate linguistic output and keep up a dialogue. At the same time, the lack of any live interactions with the students means that the system will not be able to ask further questions for clarification if it is unable to determine students' knowledge based on the material given. Furthermore, due to the informal, conversational style and the nonscripted and open-ended nature that make clinical interviews so unique and valuable a research tool, the system can make few if any assumptions about the form of the input and-as illustrated in the examples below - must be robust in the face of semantically incoherent or grammatically ill-formed utterances.

The computational method we applied in our experiments is a variant of latent semantic analysis (LSA). This 
technique has already proven to be reasonably accurate in generating measures of student knowledge as reflected in their written work (Foltz, Laham, \& Landauer, 1999; Rehder et al., 1998), and it has been used with good results in ITS development (Steinhart, 2001; Wiemer-Hastings, 2000; Wiemer-Hastings, Wiemer-Hastings, \& Graesser, 1999). It is particularly well-suited for unstructured and frequently ill-formed data of the kind we are dealing with, since it is solely based on patterns of word co-occurrence and does not presuppose any linguistic analysis, such as part-of-speech tagging or parsing.

The ultimate goal in applying computational methods to interview data is to use the linguistic output generated during interviews to retrace in detail the conceptual process that was involved in their production, identifying changes in student conceptions both during the generation of an explanation in an interview session and over larger periods of time, such as between pre- and postinstruction interviews. The immediate aim of the present study is somewhat less ambitious and more pragmatic. We are mainly interested in the use of an LSA-based method in classifying interviews and parts thereof into a small set of predefined categories. Although this ability is a necessary first step toward the ultimate goal of an automated deep analysis, it also promises to yield practical benefits in the shorter term. The time and effort required to annotate and analyze interview data manually constitute a tight bottleneck in any attempt to systematically interpret large numbers of interviews. The methods we introduce here lend themselves to application as an aid to the practicing researcher in the classification and retrieval of interview data, thus opening up the possibility of large-scale data-driven research.

In what follows, we provide an overview of some of the conceptions that students offered when asked about the causes of the seasons. We then describe the computational instrument we used to assess these conceptions and some its limitations. Finally, we report and discuss the results of our evaluation.

\section{WHAT STUDENTS THINK ABOUT THE CAUSES OF THE SEASONS}

The interview data used in this study consist of 34 transcribed responses to the question, "Why is it warm in the summer and cold in the winter?" These data had been previously collected and analyzed as part of the Conceptual Dynamics Project at Northwestern University' (more details on the process of the analysis are given below). In this section, we briefly discuss the major kinds of responses students gave in these interviews.

\section{Common Student Conceptions}

Understanding why we experience different seasons involves concepts about the earth's movements and its relationship to the sun. Many middle school students have at least some of the relevant knowledge that is required. For example, they often know that the earth is spherical, that it orbits around the sun and that the sun is a source of heat and light. However, the scientific explanation of the causes of the seasons lies outside the ken of most middle school students (Galili \& Lavrik, 1998; Trumper, 2001) and often even their teachers (Atwood \& Atwood, 1996). Among our participants $(n=21)$, only 2 students interviewed prior to instruction responded with an answer that approximates the normative explanation. On the other hand, what students do know is often enough for them to generate a coherent theory, although at times their theory is revised or completely abandoned over the course of the interview.

One way to classify the various student explanations is to decide whether they are fundamentally rooted in an explanatory principle that takes into account the distance of the earth to the sun, or one that is based on the orientation of the earth to the sun. For the practical purposes of computer assessment, we have adopted the following three general categories of student conceptions as the basis of our analysis:

1. Closer/farther misconceptions rely on the notion that changes in the distance between the earth, or parts of the earth, to the sun are responsible for the seasons.

2. Facing/not-facing misconceptions attribute the causes of the seasons to whether a particular side of the earth is facing toward the sun (i.e., summer) or facing away from the sun (i.e., winter).

3. Normative conceptions accord with the accepted scientific model that describes the main cause of the seasons (see below).

In some respects, this is an oversimplified set of possible student conceptions. A more fine-grained analysis would likely reveal a richer variety of nonnormative ideas. On the other hand, a simple three-category scheme is useful for the general classification of student conceptions. In our interviews, we found that most responses fit clearly into one of these categories. Below we provide three examples of student explanations. Each example corresponds to one of the above categories.

Jill's closer/farther explanation. When asked about the seasons, a student we named Jill described how the orbiting of the earth around the sun causes it to be closer or farther away from the sun. All students were asked to generate a drawing to support their explanation. Jill's drawing is reproduced in Figure 1. In the following transcript, Jill is providing an explanation of the seasons as she draws the earth orbiting the sun.

Here's the sun (draws a sun), and here's the earth (draws an earth and its orbital path), so this would be the summer (writes summer on drawing)... cause when the earth is closer to the sunrays hit it like closer, so it's hot.

In her drawing and in her explanation, Jill suggests that the earth's distance to the sun varies due to an off-centered orbit. This interpretation is further supported by the fact that she writes the word summer at the location on the orbit that is closest to the sun. Jill mistakenly believes that there are significant changes in distance between the earth and the sun and that these distances determine the temperatures found on earth.

Kelly's facing/not-facing explanation. Kelly develops an explanation that is based on the rotation of the earth around its axis: 


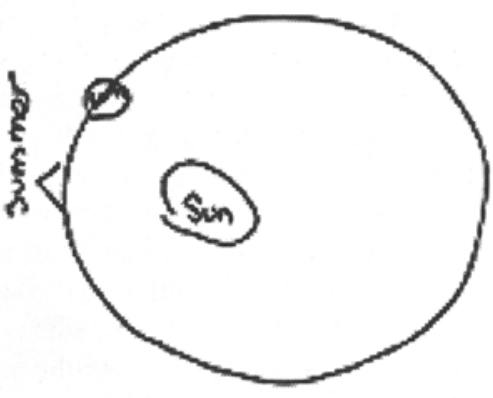

Figure 1. JPEG image of Jill's drawing of the earth's orbit around the sun.

Because when the earth is rotating and if we're having winter, the other part in like Europe or some other place ... it will be having summer, because we're facing away from the sun and they are facing toward the sun.

For Kelly, seasons are determined by whether a particular place on earth is oriented so that it is facing toward the sun or not. She believes that the side of the earth facing the sun would experience summer and the side of the earth not facing the sun would experience winter. This explanation allows her to take into account her knowledge of several of earth phenomena, including (1) that different places on earth can have different seasons simultaneously; (2) that the earth rotates on its axis; and (3) that the sun only shines on the side of the earth oriented toward the sun.

Amanda's normative explanation (postinstruction). In the following transcript, Amanda is explaining why it is colder at the poles.

the farther up or down you get (referring to different latitudes) like the more it slants ... and it [light] hits there at an angle ... it's still the same amount of light ... but it has to cover a much bigger area ... Alaska which is up here (points to her drawing) ... light hits it at more of an angle and so it has to warm up more land. It's still the same amount of light but it has to warm up more.

Amanda demonstrates that she has some knowledge of the principles that govern the seasons. She explains that at high and low latitudes, a given amount of sunlight heats a larger area of land. From this transcript we can assume that, at the very least, Amanda understands the correct relationships between the angle of sunlight striking the earth and the area warmed. There is also some evidence that she understands the effect of the varying angles of sunlight on the earth's temperature.

\section{The Normative Explanation}

The main contributing factor to the seasons is the variation in orientation of the earth to the sun as it orbits on a tilted axis. The tilt in the earth's axis causes the hemispheres to be oriented at different angles to the sun at different times of the year. When it is winter in a particular hemisphere, the axis is tilting in such a way that the hemisphere is oriented away from the sun. This causes a given amount of sunlight to be spread over a large area of land. In the summer, for either the northern or southern hemisphere, the earth's angular orientation to the sun has the effect that a given amount of sunlight warms a smaller portion of the earth's surface.

To fully grasp the main cause of the seasons, one must understand both the complex changes in orientation of the earth to the sun and how this results in the spreading or concentration of sunlight on the surface of the earth. It is not difficult to see why middle school students would have difficulties giving a scientific account of the seasons. None of the students interviewed demonstrated complete understanding of the scientific explanation, before or even after instruction. However, students' answers varied in the degree to which they included some of the scientific reasoning. For this reason, we have included a normative category in our analysis in order to measure the degree of "correctness" of student answers.

The next section describes the LSA instrument and how it was used to assess student knowledge.

\section{THE LSA INSTRUMENT}

Latent semantic analysis is a collective term for a family of related methods, all of which involve building numerical representations of textual units based on word occurrence patterns in a text corpus. Technically, these representations are vectors in a high-dimensional space. This vector space comes with a measure of distance, which by hypothesis is inversely related to semantic similarity. The success of the method in technical applications such as information retrieval and its popularity as a research tool in psychology suggest that this hypothesis holds up well for the purposes of those applications. ${ }^{2}$ Moreover, the preparatory work involved in creating these vector representations can be fully unsupervised, and once this preparatory work is done, the use of the vectors is conceptually simple and computationally efficient.

\section{WordSpace and the Infomap Algorithm}

The version of LSA that we applied in this work originated with the WordSpace paradigm developed by Schütze (1997). In this approach, it is the collocational behavior of individual words (more precisely, word types) that is encoded in the vector space. The corresponding notion of similarity is similarity in co-occurrence with other words. In this, the approach differs from many other LSA-style applications in which vectors represent documents or parts thereof (sections, paragraphs, etc.), rather than individual words. The software we used is a version of the Infomap package developed (in part by the second author) at Stanford University and freely available. ${ }^{3}$ Although some fea- 
tures used here, such as the "golist" mentioned below, are not supported by the public distribution, we will henceforth refer to our application as the Infomap system. In the remainder of this section, we describe it in some detail (see also Takayama, Flournoy, Kaufmann, \& Peters, 1999).

The basic underlying assumption is that the words with which a given word frequently co-occurs in actual text bear valuable information about its meaning (see Firth, 1957; Halliday \& Hasan, 1976; Hoey, 1991, for early articulations of this idea). To utilize such information in computational applications, each word type in the vocabulary is associated with a vector representation of the contexts in which it occurs. This information, and thus ultimately the similarity measure between words, may vary widely with the subject matter and genre of the training corpus (Kaufmann, 2000; Takayama et al., 1999). We believe that for an application like ours, a highly domain-specific training corpus is generally desirable, since it will tend to (1) contain much of the vocabulary typical of the domain; (2) give prominence to the relevant senses of ambiguous words (e.g., axis, season); and (3) yield a word similarity measure that is particularly fine-grained in the relevant lexical fields. At the same time, the corpus should not consist solely of documents adhering to a highly technical and specialized style. This is especially important in our application, since students frequently describe ideas in their own terms and should not be penalized if they merely fail to use standard terminology. We used a manually assembled corpus of public-domain texts in a variety of genres retrieved from the World Wide Web, all of which center thematically around the areas of astronomy and geology. The resulting corpus comprises about 273,000 word tokens. This is small by present-day standards, but sufficient to give surprisingly good results (see below). Owing to the idiosyncrasies of its source and the way it was obtained, the corpus consists of largely unstructured text without demarcated document boundaries. ${ }^{4}$

The word types in the training corpus are ranked by frequency of occurrence, and the Infomap system automatically selects (1) a vocabulary $W$ for which vector representations are to be collected and (2) a set $C$ of 1,000 "content-bearing" words whose occurrence or nonoccurrence is taken to be indicative of the subject matter of a given passage of text. In our implementation, these choices are guided by a stoplist of (mostly closed-class) lexical items that are to be excluded, as well as a golist of words that may be too infrequent to be included in $W$ automatically, but for which vectors are desired (in our case, this included words like axis, earth, and sun). The vocabulary $W$ consisted of 11,000 items, including all golist items and, in addition, high-frequency non-stoplist words. The set $C$ of content-bearing words contained the 50 th through 1,049th most frequent non-stoplist words. This method may seem rather blunt, but it has the advantage of not requiring any human intervention or antecedently given information about the domain.

The cells in the resulting matrix of 11,000 rows and 1,000 columns were filled with co-occurrence counts, recording, for each pair $\langle w, c\rangle \in W \times C$, the number of times a token of $c$ occurred in the context of a token of $w$ in the corpus. The context of a token $w_{i}$ in our implementation is the set of tokens in a fixed-width window from the 15th item preceding $w_{i}$ to the 15th item following it (less if a document boundary intervenes). ${ }^{5}$

The matrix was transformed by singular value decomposition (SVD), whose implementation in the Infomap system relies on the SVDPACKC package (Berry, 1992; Berry, Do, O'Brien, Krishna, \& Varadhan, 1993). The output was a reduced $11,000 \times 100$ matrix, which was then used in calculating similarity values. Thus each item $w \in W$ is associated with a 100 -dimensional vector $\mathrm{w}$, and the similarity between two words $w, v$ is measured as the cosine between the corresponding vectors: ${ }^{6}$

$$
\cos (\mathbf{w}, \mathbf{v})=\frac{\mathbf{w} \cdot \mathbf{v}}{|\mathbf{w}||\mathbf{v}|} \text {. }
$$

Vectors for multiword units of text (e.g., paragraphs, queries, or students' responses in our interview data) can be calculated as the normalized sums of the vectors associated with the words they contain. This offers a measure of flexibility that is not afforded by other LSA-style methods: ${ }^{7}$ Given a vector space obtained from a training corpus, such sum vectors can be obtained for any multiword unit, regardless of whether it occurs in the training corpus itself. Thus most crucially for our purposes, we can use the word vectors derived from our training corpus directly for calculating vector representations of students' answers in the evaluation task. This procedure is outlined in the next section.

\section{USING LSA TO ASSESS STUDENT KNOWLEDGE}

The goal of the present study was to explore the utility of LSA-style word representations in evaluating student knowledge as it is reflected in clinical interview data. To this end, we compared electronic transcripts of students' responses with text samples that are representative of the three "typical" conceptions discussed above. We will refer to these selected text samples as comparison documents. The basic method for carrying out the comparisons was already outlined in the preceding section: The vector of a document (either a student response or one of the comparison documents) is the normalized sum of the vectors of the words it contains, and based on these values, the similarity between the student's answer and each of the comparison documents is calculated as the cosine similarity between the two associated document vectors. The underlying assumption is that those comparison documents that have the highest similarity to a given student response are most representative of the content of that response.

The next two subsections outline some aspects of the materials that were common to all experiments, to which we turn subsequently.

\section{Comparison Documents}

Part of the challenge in developing an accurate classification instrument is using comparison documents that are attuned to the different student conceptions. In the intended practical application of our method as an aid in 
the analysis of large quantities of interview data, the user may calibrate the classifier by refining these documents through trial and error. However, in our study, all were produced prior to the experiments. To get a sense of what kinds of documents are most useful in the classification, we manipulated them systematically as outlined below, resulting in three versions for each. No modifications to the comparison documents were made once the evaluation had begun. In addition, no manipulations were performed on the transcripts of the student responses.

The original three comparison documents were similar in length (100 words for closer/farther, 94 for facing/not facing, and 100 for the normative explanation) and were written as coherent paragraphs, similar in style and content to the text that might appear in a scientific text on the subject.

Naturally, these documents contained many content words in common, such as earth, sun, or winter. A potential problem with common content words is that they tend to attenuate the difference between the vectors representing the documents. To counteract this effect, we produced a second version of each document by automatically removing all words that appeared in two or more comparison documents. What is left in each case is the language that is unique to a particular conception about the seasons. We refer to these as Version 2 of the comparison documents.

The third version (called Version 3) of each document was obtained by manually removing words that were judged to be irrelevant to the conception categories (e.g., the word resulting). All versions of the comparison documents are included in the Appendix.

Table 1 displays the mutual similarity values between the three documents for each of the three versions. The table shows that for each pairing, the similarity decreases with the removal of common words (Version 1 vs. Version 2), and that it further decreases in two of the pairings with the removal of irrelevant words (Version 2 vs. Version 3). This means that with each successive version, their respective locations in the vector space are farther apart.

\section{Interpretation of Interview Data}

As mentioned above, the student interviews used in this study were drawn from a larger corpus of data collected by the Conceptual Dynamics Project at Northwestern University. As part of this project, team members conducted interviews with students in the middle school grades about a range of scientific topics. The interviews were videotaped, digitized and transcribed. All of the data used in this study were drawn from the portions of the interviews in which students respond to the question: "Why is it warm in the summer and cold in the winter?" In all, we used 21

Table 1

Cosine Similarity Between Unmodified and Modified Versions of the Comparison Document Vectors

\begin{tabular}{llll}
\hline & C-CF & C-FN & CF-FN \\
\hline Version 1 & .4216 & .3573 & .7659 \\
Version 2 & .0997 & .2039 & .1740 \\
Version 3 & .0299 & .2378 & .0109 \\
\hline
\end{tabular}

Note-C, correct; CF, closer/farther; FN, facing/not facing. interviews that were taken prior to any instruction, and 13 interviews taken after instruction with a subset of the original 21 students. All portions of the electronic transcripts that represent the interviewers' words were deleted so that only the students' responses remained for analysis.

As part of the Conceptual Dynamics Project, the interview data had been manually analyzed by four independent researchers. A detailed coding scheme was developed that included 109 codes capturing various aspects of student thinking about the seasons. This coding scheme supports a much more nuanced interpretation of the interview data than is required for the purposes of this study.

Part of the process of this analysis effort was to generate written narratives that summarize the students' thinking over the course of the interviews. These summaries represent a consensus among the four researchers about each of the students' interviews. Each summary contains coarser-grained analyses that capture holistic interpretations of the data, including general categories of misconceptions that apply to each student. It is these synthesized judgments of the four annotators that were used as the objective standard in evaluating the LSA technique. The summaries do not include a record of the process by which the consensus was reached. Therefore, some potentially useful information, for instance on individual raters' confidence and interrater agreement, was not available to us.

In a similar way, changes in conception between preand postinstruction interviews, for the 13 students for which both sets of data were available, were analyzed by the four human annotators and summarized in written documents. There was consensus among the researchers that although in some cases the students' misconceptions persisted after instruction, there were, on the other hand, gains in understanding of at least some of the concepts that are part of the normative explanation of the seasons. For example, if a particular student persisted with a facing/not-facing misconception in their postinstruction interview, there may also be some discernible gain in that student's understanding of the motions of the earth in the solar system. Gains, both small and large, were observed in all students that were interviewed after instruction.

Notice that in both of these analyses - the interpretation of the preinstruction interviews and the comparison between pre- and postinstruction interviews-the judgments of the human annotators were purely qualitative. Thus although the computational method yields gradient measures of similarity, these data do not lend themselves to a correlation analysis.

\section{EXPERIMENT 1}

The goal of the first experiment was to test the accuracy of the LSA method in classifying students' preinstruction interviews into the three conceptual categories described above. The judgments of the human annotators served as the standard of comparison. From the hypothesis that a high cosine similarity between a student response and a comparison document indicates that the student expressed the corresponding conception in the interview, a classification whereby each student response is assigned the cat- 
egory that yields the highest similarity value is predicted to yield a high level of agreement with the annotators.

\section{Method}

Materials. The transcripts of 21 preinstruction interviews and the three versions of each of the three comparison documents were used.

Procedure. Document vectors were generated for all student transcripts and for the three comparison documents, and the cosine similarity was calculated for each of the student responses and each of the comparison documents. Each student response was assigned to the conceptual category whose representative comparison document yielded the highest cosine similarity value. These assignments were compared against the judgments of the human annotators. This process was repeated for each of the three versions of the comparison documents.

There were no cases of ties for the highest similarity value. In a fully unsupervised implementation of the method, the simplest action to take in case of a tie would be to assign one of the maximally similar categories at random. However, ties may arise for different reasons, thus a more useful approach in both the development and the practical application of the tool would be to flag the document as belonging to an "other" category and inspect it manually. For instance, a tie might suggest refinements or revisions of the categories and/or the comparison documents. A tie might also indicate a change in the student's response in mid-interview, for instance from closer/ farther explanation early on to the normatively correct explanation in the latter part. Some examples in which such shifts occurred in our data are discussed in Experiment 3 below.

\section{Results}

The classification using the full versions of the comparison documents was in agreement with the annotators in 14 of the 21 cases, or $66 \%$. A $\chi^{2}$ test of independence was performed to examine the relation between human and LSA assignments. These variables are significantly associated $\left[\chi^{2}(2, N=21)=16.26, p=.0026\right]$. For Versions 2 and 3 , agreement with the annotators rose to 17 $(81 \%)\left[\chi^{2}=23.62, p=.00001\right]$ and $19(90 \%)\left[\chi^{2}=\right.$ $29.61, p=5 \mathrm{E}-06]$, respectively. These results are summarized in Figures 2-4. There was no systematic correlation between the length of the interviews (ranging from 54 to 957 words) and the cosine values. Correlations between lengths and the cosine values were obtained using the third versions of the normative $[r(21)=.15]$, the facing/not facing $(r=.46)$, and the closer/farther $(r=-.38)$ comparison documents.

\section{Discussion}

The classification by the instrument shows considerable agreement with the judgments of the human annotators, especially with Versions 2 and 3 of the comparison documents. To underscore this point, consider two possible baseline assumptions. First, a random assignment of categories to each interview would yield an expected accuracy of $33 \%$. Second, an assignment of the most frequent category would yield a higher accuracy, but still be outperformed by our system. The most frequent category is "closer/farther," assigned by the annotators to 9 interviews (43\%), or to 11 interviews (52\%) if one includes 2 interviews that were judged equally representative of the closer/farther and facing/not-facing misconceptions. Both of these are outperformed by our system on all three versions of the comparison documents, especially on Versions 2 and 3.

Given the simplicity of our "winner-takes-all" categorization algorithm, this performance is impressive. A more

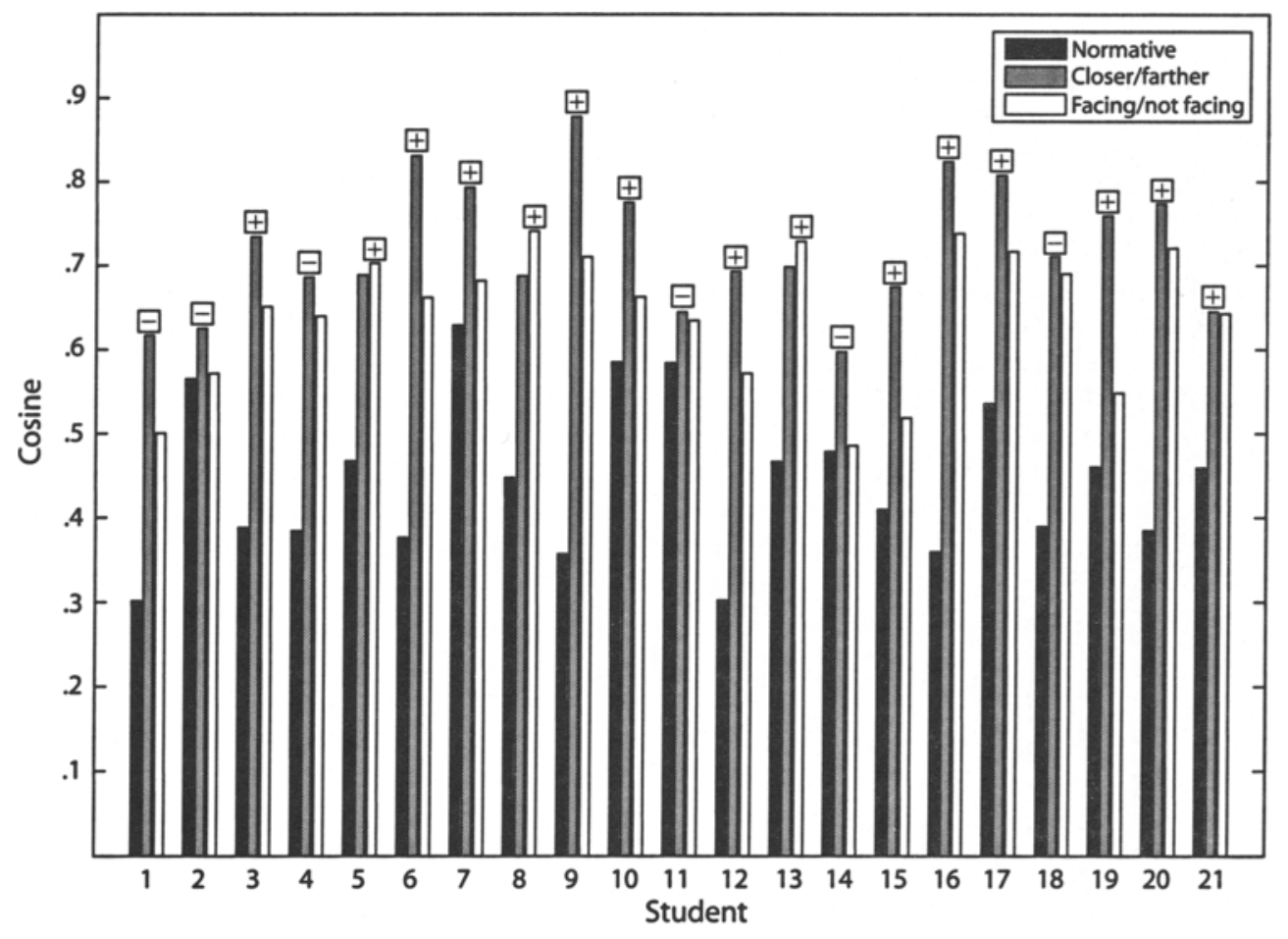

Figure 2. Version 1 comparison document classification results. Plus (+) and minus ( - ) signs indicate correct classification and misclassification, respectively, as compared with human judgments. 


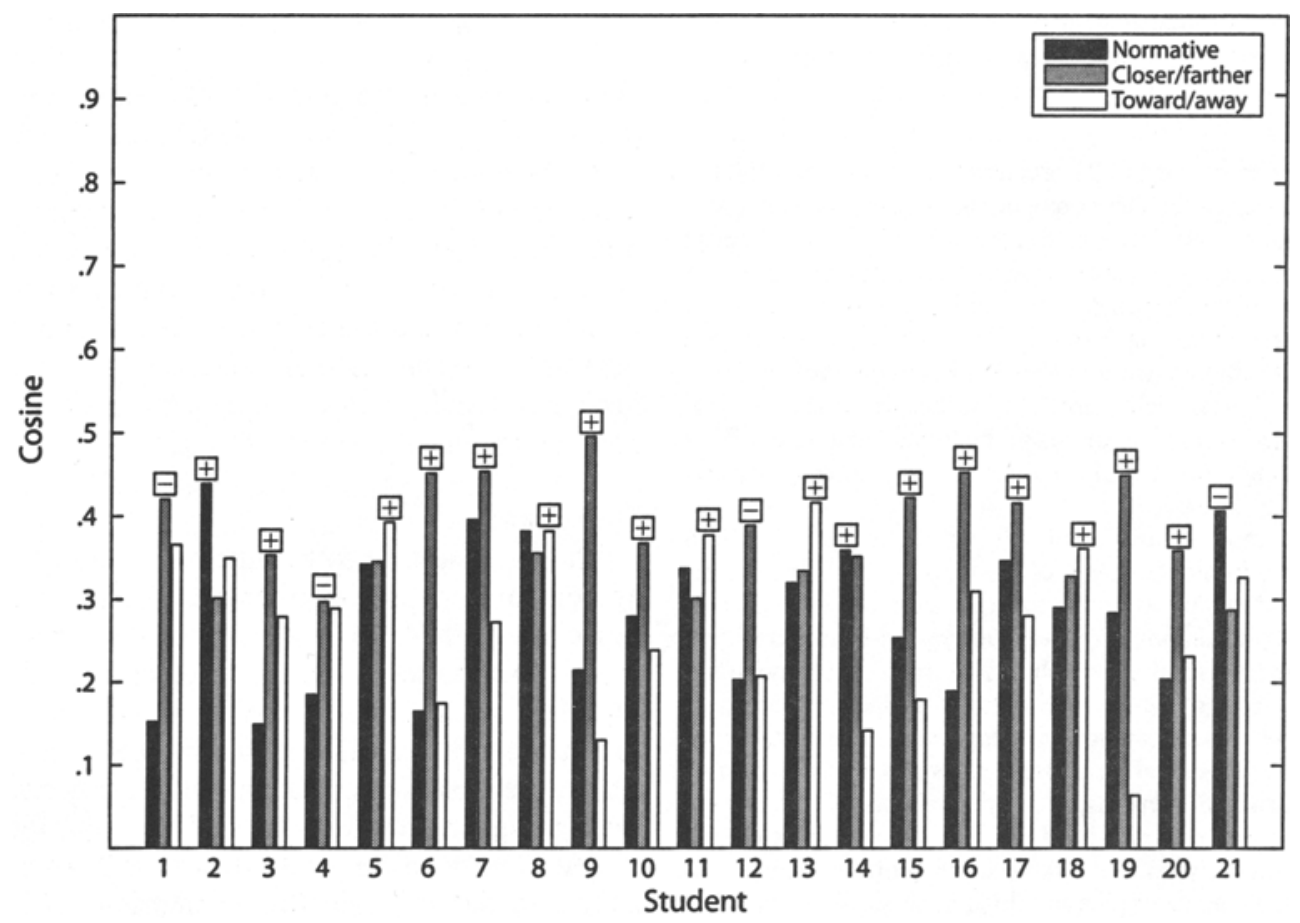

Figure 3. Version 2 comparison document classification results. See Figure 2 caption for more details.

nuanced analysis might provide further insights into the method's strengths and weaknesses and suggest ways in which it could be improved. For instance, the relative ranking of the two "losing" categories might also provide useful information about the accuracy of the algorithm. However, an objective evaluation by these criteria was not feasible because the human raters' judgments at our disposal did not include the relevant information, such as confidence scores or the opinions of individual raters. We leave such a more fine-grained evaluation for future work.

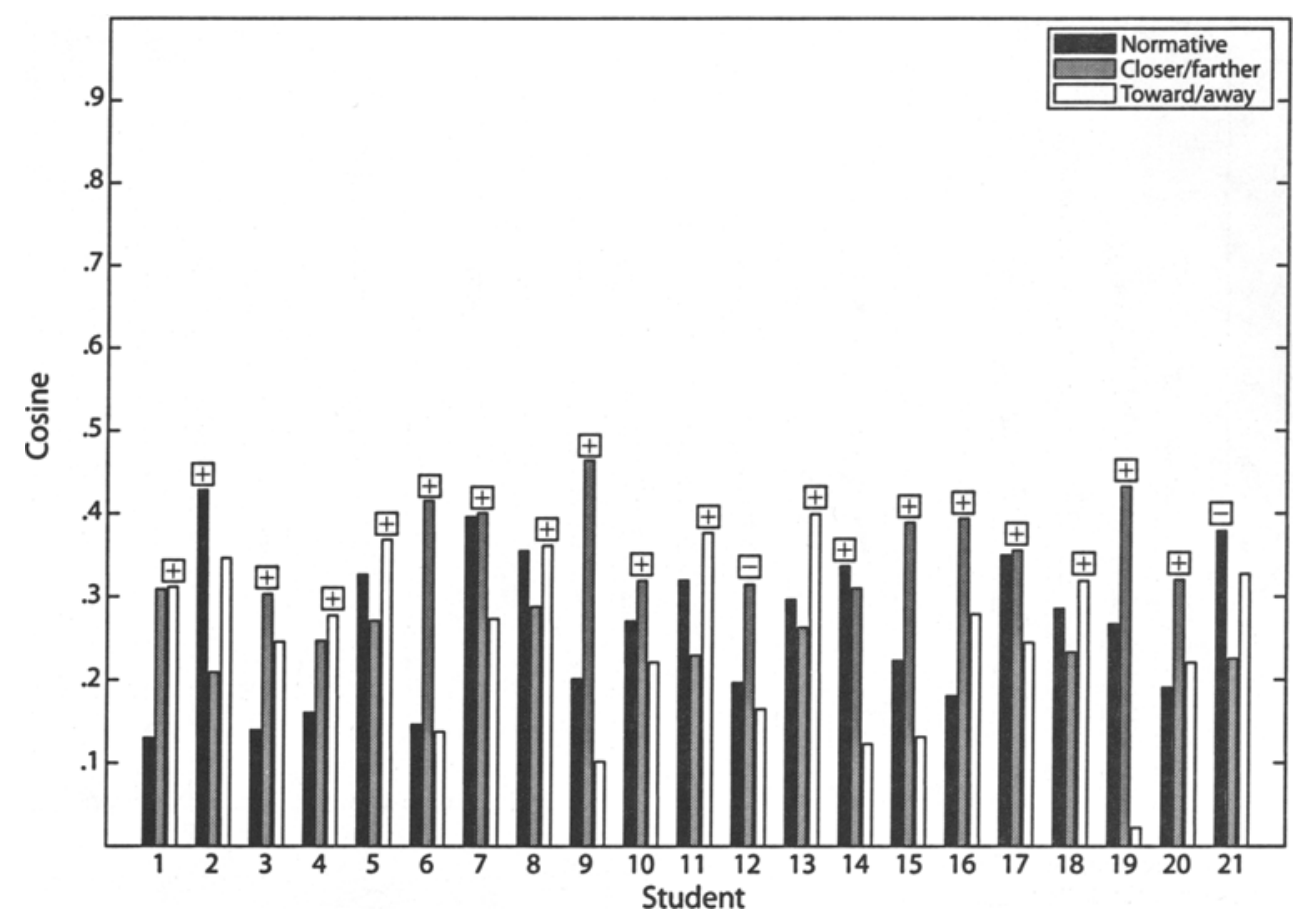

Figure 4. Version 3 comparison document classification results. See Figure 2 caption for more details. 


\section{EXPERIMENT 2}

In the second experiment, the LSA tool was applied to assess changes in students' responses between pre- and postinstruction interviews.

\section{Method}

Materials. The transcripts of 13 postinstruction interviews and Version 2 of the comparison document vectors obtained in Experiment 1 were used.

Procedure. Document vectors were generated for all 13 postinstruction transcripts. Cosine similarity values between the postinstruction interviews and the comparison documents were calculated and compared with those of the preinstruction interviews for the same students. The differences were compared with the human judgments regarding the students' changes in conceptions.

\section{Results}

The mean changes in cosine values for the 13 students are illustrated in Figure 5. Every postinstruction interview showed an increase in cosine similarity to the normative comparison document (solid line) $[t(26)=-3.89, p=$ $.0007]$. Nine of the 13 postinstruction interviews had a lower cosine value for the closer-farther misconception than in the corresponding preinstruction interviews (dashed-dotted line). Eight of the students' cosine values for the facing/not-facing misconception decreased in the postinstruction interviews (dotted line). However, no statistically significant differences were found between the cosine values of the pre- and postinterviews with respect to the two misconception categories: facing/not facing $(t=0.22, p>.82$, n.s. $)$ and closer/farther $(t=1.13, p>$ .26, n.s.).

\section{Discussion}

The instrument's evaluations of the changes in conceptions from pre- to postinstruction interviews agree well with the human analyses described in the Interpretation of Interview Data section. There is a measurable increase in the semantic similarity between the postinterviews and the

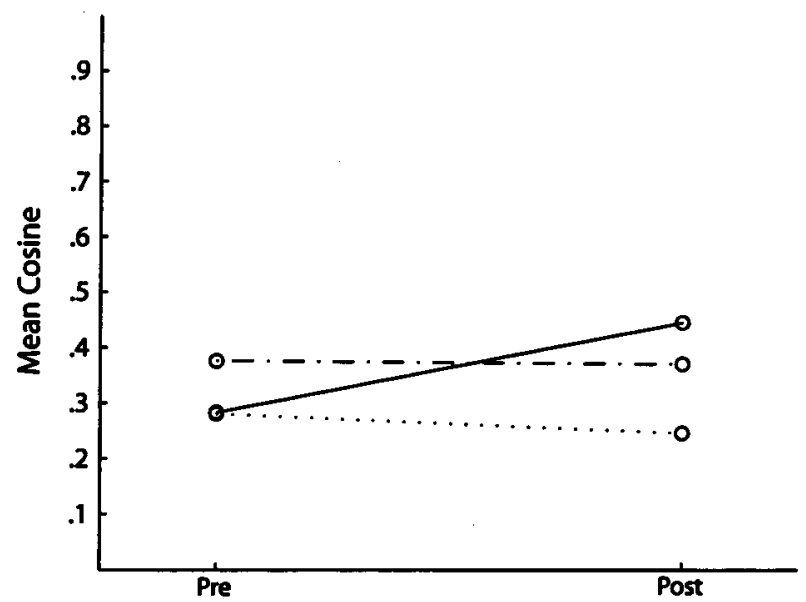

Figure 5. Changes in mean cosines of 13 students from pre- to postinstruction interviews. Each line represents one of the three conception categories (normative $=$ solid line, closer/farther $=$ dashed-dotted line, and facing/not facing $=$ dotted line). normative document for all 13 students. A minimal interpretation of these results would be that students began to use more of the relevant vocabulary found in the normative comparison document when interviewed after instruction. This may reflect an increase in understanding of some the appropriate concepts. However, the cosine values for the misconceptions declined only slightly for most students and in some cases even increased between pre- and postinstruction interviews. This seems to reflect the annotator's assessments, who reported that many of the students persisted in their misconceptions after instruction.

\section{EXPERIMENT 3}

The purpose of the third experiment was to assess the instrument's capacity to correctly identify changes in conceptions that occur within a single interview. As we noted in the introduction, one of the applications in which an LSA tool could prove useful is in detecting and tracking conceptual changes not just between, but also within interviews. Although the present study was not specifically concerned with this application, our interview data offered an opportunity for some exploratory work in this direction. Specifically, the human annotators had found evidence that two of the students had changed their minds regarding what causes the seasons during the course of the interview. The students we named Edgar and Kelly answered the interviewer's question by first providing an explanation that was consistent with one of the two misconception categories, and later shifted to an alternative explanation consistent with the other misconception. In this experiment, we tested whether our instrument is able trace these changes by splitting the interviews into smaller parts and categorizing each of those segments.

\section{Method}

Materials. The preinstruction interview transcripts of Edgar and Kelly, and the three versions of the comparison document vectors were used.

Procedure. Kelly and Edgar's transcripts were divided into a series of 50 word segments and a remainder segment. Kelly's transcript, which is composed of a total of 262 words, was consequently sectioned into four segments of 50 words and one segment of 62 words. Edgar's transcript was divided into five segments of 50 words each and a remaining segment of 58 words. The selection of the segment size was based on an estimation of how large a portion of a typical interview can include something like a coherent conception. Document vectors were generated for each of the segments of the transcripts. Cosine similarity values were then obtained to measure the similarity between the segments and each of the misconception comparison documents.

\section{Results}

The results are shown in Figure 6 for Edgar's interview and in Figure 7 for Kelly's, for each of the three version of the comparison documents. For both students, the graphs show the similarity between each of the segments and the comparison documents for the closer/farther misconception (solid line) and the facing/not-facing misconception (dashed line). As is evident from the graphs, in both cases there is a change during the interview in the comparison document with the highest similarity value. In Edgar's in- 
terview, Segments 1 and 2 exhibit the highest similarity to the facing/not-facing comparison document. In contrast, Segments 4 and 5 are most similar to the closer/farther document. The change in Kelly's case is in the opposite direction: The cosine value for Segment 1 is higher for the closer/farther misconception, whereas Segment 5 is more similar to the facing/not-facing misconception. Notice that this change is amplified in Versions 2 and 3 of the comparison documents.

\section{Discussion}

The transitions in the similarity values may be interpreted as indicating conceptual change in the course of the interview. This conclusion was corroborated by the qualitative assessments of the human annotators. For illustration, we reproduce here some of the passages from each interview. First, the following are portions of Edgar's interview. In Segment 2, he presents a facing/not-facing explanation. Segment 5 , on the other hand, shows a closer/ farther conception:

Segment 2: "the rays are hitting directly right here (points to side of the earth facing the sun) so things are getting hotter in the summer and when this thing turns the country will be here (points to side of the earth not facing the sun) and the sun can't reach as much"

Segment 5: "It's like summer right here cause its actually closer to the sun than over here (points to the opposite end of the orbit) so when it moves ... it reaches here and it's further away from the sun."

The shift in Kelly's explanation in the opposite direction is illustrated in the following passages. Segment 1 is evidence for the closer/farther conception:

Segment 1: "Because when the Earth is at summer the Earth is more close to the sun and in the winter it is far to the sun because the Earth orbit is not a exact circle."

Later on, in Segment 5, Kelly explains that Europe experiences summer when it is facing the sun and North America is facing away from the sun:
Segment 5: "[Europe is] having summer because we're facing away from the sun and they [Europe] are facing toward the sun."

These results provide some evidence that the instrument's assessments are sensitive to the conceptual content contained in small portions of transcripts. The changes in cosine similarity correspond to the students' shifts in explanation as observed by the annotators. However, ultimately the goal in applying the LSA tool to the analysis of conceptual changes during interviews is to detect such shifts, rather than merely tracing them in those transcripts of which it is known that changes are attested. At present, we are uncertain as to how accurately our tool could detect changes. An analysis by segments similar to that for Edgar and Kelly was carried out on all other interviews and, not surprisingly, showed some degree of fluctuation in cosine values for all of them. An automatic method for finding interviews that exhibit conceptual changes seems feasible, but would likely return many false positives. A systematic evaluation would be problematic, since the human annotators did not clearly indicate of all interviews whether or to what extent conceptual shifts were taking place. Thus in conclusion, the evidence offered by Experiment 3 is anecdotal, but suggestive of promising directions for future work.

\section{GENERAL DISCUSSION AND CONCLUSION}

The three experiments reported above tested our LSAbased technique on three basic tasks: the classification of student interviews according to a coarse-grained, threeway taxonomy of typical responses; the comparison of pre- and postinstruction interviews to assess differences brought about by the instruction; and the classification of smaller passages to detect conceptual change during the course of the interview itself. The instrument performed well on all three of these tasks, as measured by its agreement with human annotators. The results are encouraging and suggest that the method is potentially useful in educational research in a number of ways. In this final section, we discuss some possible applications, limitations, and directions for future development.
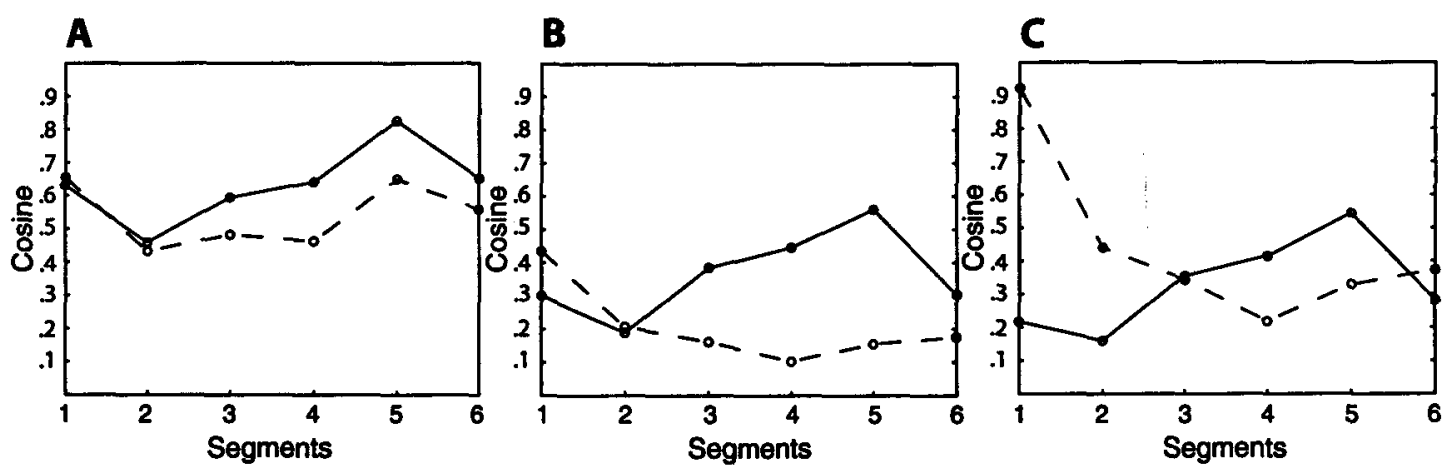

Figure 6. Cosine similarity values between each segment of Edgar's transcript and the comparison documents for the closer/farther misconception (solid line) and the facing/not-facing misconception (dashed line). Panels A, B, and C represent cosine values for Versions 1, 2, and 3 of the comparison documents, respectively. 

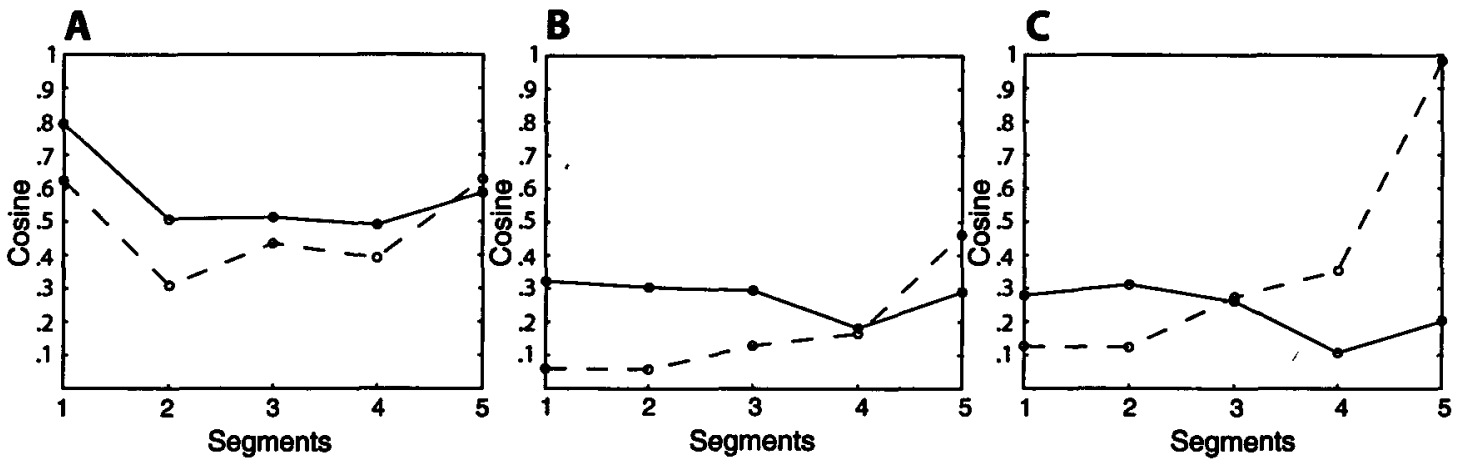

Figure 7. Cosine values of Kelly's transcript segments. Panels A, B, and C represent cosine values for Versions 1, 2, and 3 of the comparison documents, respectively.

The most promising practical application of the method at its present stage of development would seem to be as an aid in the management and annotation of interview data. Considering the time-consuming nature of the analysis, even a relatively coarse-grained classifier like ours can be helpful in preprocessing, coding, indexing and retrieving transcribed interview transcripts, thus supporting research on data collections on a larger scale than what is now feasible. It could easily be ported to different domains or even other languages; a moderately sized training corpus and good comparison documents are all that is needed. Within the Conceptual Dynamics Project at Northwestern University, from which the transcripts used in our study were drawn, we plan to develop and test a prototype of such a data management system as the project scales up to larger and thematically more diverse data sets.

In the long run, however, the goal is to develop computational tools that play more than a supporting role in the analysis of interview data. Although it seems to us neither realistic nor even desirable to try to eliminate the human judge altogether, we envision a future version of the computational tool which will annotate interview data at a much finer grain and in a largely unsupervised fashion, reducing the job of the human user to little more than quality control. As of now, this is a distant goal and the scope of our study is modest in comparison. But it is worthwhile to consider the directions in which future work ought to proceed, as well as some of the pitfalls to be aware of in the process.

One issue concerns the composition of the training corpus used in deriving the word vector representations. A potential difficulty with the automated analysis of student interviews is the varied and nontechnical vocabulary which participants use in their responses. As we mentioned above, this challenge is shared with other applications, such as ITSs, but it is especially serious in our domain, given the unscripted character of the clinical interviews and the noninteractive nature of the transcripts. The focus on a particular subject matter alleviates the problem somewhat, but even so, the idiosyncratic word usage of the interviewees is a potential stumbling block for any computer assessment. In order to circumvent it, the vocabulary represented in the vector space should be large enough to contain most of the possible lexical choices, and it must correctly represent the semantic relationships between the words. It turned out helpful in this respect that we used a training corpus containing a range of different styles and genres, which yielded word vectors for most of the content words that students used in our data (recall that the training corpus did not contain the interview data). There is some evidence that as long as all words are represented, the classification is fairly robust under variation in lexical choices. For example, a student we called Mark consistently used the word slant to describe the tilt of the earth's axis. Our vocabulary contains the word slant but did not rank it among the 20 most similar words to tilt. (The word slanted also occurred and was ranked 14th most similar to tilt.) To see whether Mark's idiosyncratic use affected the evaluation of his interview, we substituted all occurrences of slant or its inflectional forms with the corresponding forms of tilt and submitted the edited version of his interview to the same procedure as that for Experiment 1. We found that the effect of this substitution on the similarity was minimal and did not change the qualitative classification of Mark's interview. His transcript was deemed "correct" (i.e., giving the normative explanation) by the human annotators. Both with and without the substitution of tilt for slant, his transcript was incorrectly categorized as "closer/farther" with Version 1 of the comparison documents, but correctly with both Versions 2 and 3 of the comparison documents. The numbers are given in Table 2 .

Notice that, not surprisingly, there seems to be a general trend toward higher scores for the normatively correct response after the substitution. What is interesting is that without the substitution, the numbers are very similar to those obtained with the substitution. This is in line with the fact that LSA generally performs well in applications where word similarity is more important than perfect string match (e.g., information retrieval).

Whether such a substitution of synonyms could be performed automatically is a separate question. The cooccurrence based notion of "similarity" captured by LSAstyle vector representations correlates with a variety of semantic relations, including antonymy, and invariably includes some amount of unexplained noise, especially with 
Table 2

Mark's Cosine Similarity With the Comparison Documents With (Left) and Without (Right) Substitution of Tilt for Slant

\begin{tabular}{lccccccc}
\hline & \multicolumn{3}{c}{ Without Substitution } & & \multicolumn{3}{c}{ With Substitution } \\
\cline { 2 - 3 } \cline { 6 - 8 } & C & CF & FN & & C & CF & FN \\
\hline Version 1 & .4788 & .5972 & .4857 & & .4761 & .5707 & .4530 \\
Version 2 & .3589 & .3513 & .1417 & & .4254 & .3319 & .1172 \\
Version 3 & .3364 & .3098 & .1225 & & .3931 & .2541 & .0957 \\
\hline
\end{tabular}

Note-C, correct; CF, closer/farther; FN, facing/not facing.

a small training corpus which may lack the sheer numbers to overwhelm accidental features of small sets of occurrences. For instance, the 20 nearest neighbors of the word tilt in our training data are listed in Table 3. Although a few of these can be said to be more or less directly related to the subject matter of the interviews, most do not stand in a clear relationship to tilt.

Thus, the fact that slant can safely be substituted for tilt in interviews in our collection cannot be automatically read off the geometry of the vector space. If an automatic substitution of this kind is to succeed at all, it will have to rely on additional sources of information. One promising direction in this respect may be a combination of LSA with a hand-coded lexical knowledge base such as WordNet (Fellbaum, 1998), in which a variety of semantic relationships are explicitly labeled. We leave such an extension to future work.

Aside from the inability to distinguish linguistically interesting semantic relationships from accidental ones, LSA-style applications in general are afflicted with an even more serious weakness: The only information about words that is utilized comes from patterns of co-occurrence, with no regard for syntactic relations among the words or nontrivial linguistic structure of any other kind. As a result, although LSA can detect what topic a given passage is about, it usually cannot determine what exactly is asserted about that topic. Therefore, not only could it potentially give high scores to students who use the correct vocabulary to make false assertions, but it would not even distinguish meaningful prose from simple lists of keywords. In general, the use of the method is only sound to the extent that the use of the relevant words is in fact indicative of a corresponding understanding of the concepts behind them and the way those concepts are related. This assumption is relatively safe in our research context, but it would be highly problematic

Table 3

The 20 Nearest Neighbors of the Word Tilt, With Their Cosine Values

\begin{tabular}{lcll}
\hline \multicolumn{1}{c}{ String } & Cosine & \multicolumn{1}{c}{ String } & Cosine \\
\hline Tilt & 1.000000 & Timing & .326511 \\
Obliquity & .458158 & Statements & .315499 \\
Earth's & .418131 & Seasonality & .313253 \\
Tilted & .373762 & Likened & .313175 \\
Inclination & .366088 & Slanted & .303560 \\
Rotation & .347975 & Observation & .303319 \\
Moderation & .340148 & Assemble & .300740 \\
Car & .335777 & Peter & .298739 \\
Pogge & .328377 & Denver & .297786 \\
Richard & .328377 & Thurimella & .297786 \\
\hline
\end{tabular}

in actual teaching settings, in which students might easily figure out how to "game" the system. Especially in such practical applications, it seems unreasonable to expect an LSA-based system to function without human supervision until considerable progress has been made toward integrating LSA with deep linguistic analysis to arrive at a true understanding of the text. In the analysis of clinical interviews, which as we mentioned above frequently contain fragmentary and grammatically ill-formed speech, such progress seems particularly far off. Meanwhile, it is important to be aware of this shortcoming, especially in applying the method to assess content that depends heavily on its syntactic structure for its correct interpretation.

Related to the previous point, careful consideration must be taken when deciding what kinds of conclusions can be drawn from the interviews. For example, in Experiment 3, it is tempting to conclude that Kelly has changed her mind mid-interview and that her last explanation is most representative of her beliefs. However, there is evidence in the transcripts that Kelly is simply trying out a number of ideas. From her interview, it seems that she is not convinced that any of her explanations are correct. Again, we conclude that interpretations about what the computational similarity measurements represent must be appropriately supported by human assessments that are informed by the context and intentions of the participants' statements.

In sum, with careful consideration of the instruments' limitations, it is possible to obtain useful measurements that can help expedite the task of interpreting interview transcripts. For instance, the capacity to assess small portions of interviews allows for the detection of shifts in conceptual content within an interview. The promise of this approach lies in its potential ability to render more informative interpretations of interviews than methods that focus on holistic categorization or scoring. It may also be useful for researchers interested in acquiring statistics on the frequency of occurrence of particular content found in large sets of interviews. However, special care must be taken not to misconstrue the analyses of the instrument. At present, the most promising and technologically feasible application of the instrument would be as a supplemental tool in the analysis of interview data.

\section{AUTHOR NOTE}

We thank Bruce Sherin for providing us with the data and for many helpful discussions and comments, as well as Spyros Konstantopoulos and an anonymous reviewer. Correspondence relating to this article may be sent to G. Dam, Northwestern University, Onterie Center, Rm 931, 441 E. Erie St., Chicago, IL 60611 (e-mail: g-dam@northwestern.edu).

\section{REFERENCES}

ATwoop, R. K., \& ATwoon, V. A. (1996). Preservice elementary teachers' conceptions of the causes of seasons. Journal of Research in Science Teaching, 33, 553-563.

BERRY, M. (1992). Large-scale singular value computations. International Journal of Supercomputer Applications, 6, 13-49.

BerRy, M., DO, T., O'Brien, G., Krishna, V., \& VARADHaN, S. (1993). SVDPACKC (Version 1.0) user's guide (Tech. Rep. UT-CS-93-194). Knoxville, TN: University of Tennessee, Department of Computer Science. 
CAREY, S. (1988). Recognition of knowledge in the course of acquisition. In S. Strauss (Ed.), Ontogeny, phylogeny, and historical development (pp. 1-27). Norwood, NJ: Ablex.

Fellbaum, C. (1998). WordNet: An electronic lexical database. Cambridge, MA: MIT Press.

FIRTH, J. R. (1957). A synopsis of linguistic theory 1930-1955. In Studies in linguistic analysis (pp. 1-32). Ox ford: Blackwell.

Foltz, P. W., LAHAM, D., \& LANDAUER, T. K. (1999). The Intelligent Essay Assessor: Applications to educational technology. Interactive Multimedia Electronic Journal of Computer-Enhanced Learning, 1. Retrieved from www.knowledge-technologies.com.

GALILI, I., \& LAVRIK, V. (1998). Flux concept in learning about light: A critique of the present situation. Science Education, 82, 519-613.

GINSBURG, H. P. (1997). Entering the child's mind: The clinical interview in psychological research and practice. New York: Cambridge University Press.

Graesser, A. C., Lu, S. L., Jackson, G. T., Mitchell, H. H., VenTURA, M., OlNeY, A., \& LouWerse, M. M. (2004). AutoTutor: A tutor with dialogue in natural language. Behavior Research Methods, Instruments, \& Computers, 36, 180-192.

Halliday, M. A., \& HASAN, R. (1976). Cohesion in English. London: Longman.

HoEY, M. (1991). Patterns of lexis in text. New York: Oxford University Press.

Kaufmann, S. (2000). Second-order cohesion. Computational Intelligence, 16, 511-524.

MANNING, C., \& SCHUUTZE, H. (2002). Foundations of statistical natural language processing. Cambridge, MA: MIT Press.

Posner, G. J., Strike, K. A., Hewson, P. W., \& GerTzog, W. A. (1982). Accommodation of a scientific conception: Toward a theory of conceptual change. Science Education, 66, 221-227.

ReHDer, B., Schreiner, M. E., Wolfe, M. B., Laham, D., LANDauer, T. K., \& KINTSCH, W. (1998). Using Latent Semantic Analysis to assess knowledge: Some technical considerations. Discourse Processes, $25,337-354$.

SCHÜTZE, H. (1997). Ambiguity in language learning: Computational and cognitive models. Stanford, CA: CSLI Publications.

SteINHART, D. (2001). Summary Street: An intelligent tutoring system for improving student writing through the use of latent semantic analysis. Unpublished doctoral dissertation, University of Colorado, Boulder.

Takayama, Y., Flournoy, R., Kaufmann, S., \& Peters, S. (1999). Information retrieval based on domain-specific word associations. In N. Cercone \& K. Naruedomkul (Eds.), Proceedings of the Pacific Association for Computational Linguistics (PACLING '99) (pp. 155161). Waterloo, ON, Canada.

Trumper, R. (2001). A cross-age study of junior high school students' conceptions of basic astronomy concepts. International Journal of Science Education, 23, 1111-1123.

WADE-STEIN, D., \& KINTSCH, E. (2004). Summary Street: Interactive computer support for writing. Cognition \& Instruction, 22, 333-362.

WIEMER-Hastings, P. (2000). Adding syntactic information to LSA. In L. R. Gleitman \& A. K. Joshi (Eds.), Proceedings of the Twenty-Second Annual Conference of the Cognitive Science Society (pp. 989-993). Mahwah, NJ: Erlbaum.

Wiemer-Hastings, P., Wiemer-Hastings, K., \& Graesser, A. (1999). Improving an intelligent tutor's comprehension of students with latent semantic analysis. In Al in Education 1999, Le Mans, France, July 19-23, 1999 (pp. 535-542). Amsterdam: IOS Press.

\section{NOTES}

1. conceptual-dynamics.sesp.northwestern.edu/project.html.

2. The popularity of LSA stems largely from its success in practical applications. On the other hand, most theoretical linguists would argue that the relationship between similarity in collocational behavior and semantic similarity is not well enough understood to warrant the label "semantic analysis."

3. infomap-nlp.sourceforge.net.

4. The corpus, the stoplist and the golist used in this work are available upon request.

5. Two details are glossed over here: First, the Infomap system weighs this raw count with a tf.idf measure of the column label $c$. The term tf.idf stands for the product of term frequency and inverse document frequency. The formula used is the following: $t f i d f(c)=t f(c) \times$ $\{\log (D+1)-\log [d f(c)]\}$, where $t f$ and $d f$ are the number of occurrences of $c$ and the number of documents in which $c$ occurs, respectively, and $D$ is the total number of documents. Second, the number in each cell is replaced with its square root, in order to approximate a normal distribution of counts and attenuate the potentially distorting influence of high base frequencies.

6. For $n$-dimensional vectors $\mathrm{w}, \mathrm{v}$, the inner product or dot product is defined as

$$
\mathbf{w} \cdot \mathbf{v}=\sum_{i=1}^{n} \mathbf{w}_{i} \mathbf{v}_{i}
$$

The length of $w$ is

$$
|\mathbf{w}|=\sqrt{\mathbf{w} \cdot \mathbf{w}}
$$

7. LSI and some variants of LSA build the vector space from worddocument co-occurrence counts. The vector spaces derived via this method are not as easily portable. 


\begin{abstract}
APPENDIX
Comparison Documents: Text of the Three Versions Used As Comparison Documents for Each Category

\section{Normative Comparison Documents}

\section{Version 1}

The Earth's axis is tilted from perpendicular. Since the axis is tilted, different parts of the globe are oriented toward the Sun at different times of the year. Summer is warmer than winter because the Sun's rays hit the Earth at a more direct angle during summer than during winter. The light rays that hit the surface straight on, warm a smaller area of the surface of the earth. Light rays that strike the earth at an angle have to warm up a larger surface of the earth, which means that the light is spread out over a larger area.
\end{abstract}

\title{
Version 2
}

axis tilted perpendicular Since axis tilted different parts globe oriented different year because direct angle during during surface straight smaller area surface strike angle have up larger surface means spread out over larger area

\section{Version 3}

axis tilted perpendicular axis tilted oriented direct angle surface straight smaller area surface strike angle larger surface spread out over larger area

\section{Version 1}

\section{Toward/Away Comparison Documents}

The Earth orbits around the sun. Sometimes one side of the earth is facing the sun and that side experiences summer. At times the side of the earth we are on is not facing the sun and therefore we experience winter. As the earth moves around the sun the heat from the sunlight is hitting the side that is towards the sun. The side that is not facing the sun experiences winter. When the earth is towards the sun it is hotter, when the sun is facing away from the sun it is colder.

\section{Version 2}

Sometimes one side facing side experiences side we not facing therefore we experience side towards side not facing experiences toward facing

\section{Version 3}

side facing side side not facing side toward side not facing towards facing

\section{Version 1}

\section{Closer/Farther Comparison Documents}

The seasons are caused by the fact that the earth moves in an orbit that goes closer to the sun in the summer and the farther away from the sun in the winter. When the earth rotates orbits around the sun it gets closer and further away resulting in more or less sun sunlight sunrays heat temperature. The closer the earth is to the sun the hotter it is. The farther away the earth is from the sun the colder it is. The light rays must travel a longer distance in the winter and a shorter distance in the winter.

\section{Version 2}

seasons caused fact goes closer farther rotates gets closer further resulting or less sunrays temperature closer farther must travel longer distance shorter distance

\section{Version 3}

closer farther rotates closer further less sunrays temperature closer farther travel longer distance shorter distance 